\title{
RECENT DEVELOPMENTS ON A NONLOCAL PROBLEM ARISING IN THE MICRO-ELECTRO MECHANICAL SYSTEM
}

\author{
JONG-SHENQ GUO
}

\begin{abstract}
In this paper, we study an evolution problem arises in the study of MEMS (micro-electro mechanical system) device. We consider both parabolic and hyperbolic type problems. We summarize some recent results on the steady states and the global vs non-global existence of solutions. We also list some open problems and provide a list (far from complete) of references related to this subject.
\end{abstract}

\section{Description of the model: MEMS}

We are interested in the dynamic deflection of an elastic membrane inside a micro-electro mechanical system (MEMS). Typically, the MEMS device consists of an electric membrane hanged above a rigid ground plate, connected in series with a fixed voltage source and a fixed capacitor. We refer to $[29,30]$ and the references therein for the physical backgrounds and its applications.

In the case the distance between the plate and the membrane is relative small compared to the length of the device, the original mathematical system describing the operation of the MEMS is reduced to the following single nonlocal equation in dimensionless variables

$$
\varepsilon u_{t t}+u_{t}=\Delta u+\frac{\lambda f(x)}{(1-u)^{2}\left(1+\alpha \int_{\Omega} \frac{1}{1-u} d x\right)^{2}} \text { in } \Omega,
$$

where $\Omega$ is the domain of the plate and the gap between the membrane and the plate on the boundary is normalized to be 1 .

In (1.1), $u$ is the deflection of the membrane, $\varepsilon$ is the ratio of the interaction due to the inertial and damping terms in the model, while $\lambda \propto V^{2}$ in which $V$ stands for the applied voltage, and the nonnegative function $f(x)$ represents varying dielectric properties of the membrane. The integral in the equation (1.1) arises due to the fact that the device is embedded

Received May 5, 2014, accepted May 6, 2014.

This work was partially supported by the National Science Council of the Republic of China under the grant NSC 102-2115-M-032-003-MY3. This work is written during a visit of the author in April, 2014, to the Center for PDE, ECNU, Shanghai, China. The author would like to express his thanks for the warm hospitality. Also, the author would like to thank Professor Fang Li for the valuable comments. 
in an electrical circuit with a capacitor of fixed capacitance. The parameter $\alpha$ denotes the ratio of this fixed capacitance to a reference capacitance of the device. The limiting case $\alpha=0$ corresponds to the case where there is no capacitor in the circuit.

Some physically suggested dielectric profiles are the power-law profile

$$
f(x)=|x|^{p}, p>0
$$

and the exponential-law profile

$$
f(x)=e^{k\left(|x|^{2}-c\right)}, k, c>0 .
$$

Since the edge of the membrane is kept fixed, we have the boundary condition

$$
u=0 \quad \text { on } \quad \partial \Omega \text {. }
$$

Therefore, our model is the problem for (1.1) and (1.2) supplemented by a suitable initial condition(s).

We refer to $[4,5,6,9,11,12,21,22,23,24,26,27]$ (far from complete) for some recent studies on MEMS.

We shall concentrate on the case when the device is with capacitor and constant $f$. Without loss of generality, we may assume that $f \equiv 1$. We shall consider both the parabolic case (with $\varepsilon=0$ ) and the hyperbolic case (with $\varepsilon=1$ after a rescaling).

When the solution $u(x, t)$ of the initial boundary value problem for (1.1) reaches the value 1 at some point of $\Omega$ in finite time, we say that $u$ quenches in finite time. In this case, the solution creates a singularity due to that the source term in the right-hand side of (1.1) becomes infinite. Physically, this corresponds to the phenomenon of touch-down in which the membrane touches the ground electrode plate. In real applications, this touch-down phenomenon is observed when the applied voltage $V$ exceeds a fixed value.

This paper is organized as follows. In section 2, we provide some quenching criteria for both problems with and without inertia. In section 3 , we study the steady states. Then we give some global existence theorems in section 4. Finally, some open problems are stated in section 5 .

\section{Quenching criteria}

In this section, we provide some quenching criteria for both parabolic and hyperbolic problems. 


\subsection{Without inertia $(\varepsilon=0)$ : parabolic case}

In this subsection, we assume that $\alpha=1$ (after a rescaling). Hence our problem becomes (P):

$$
\begin{cases}u_{t}=\Delta u+\frac{\lambda}{(1-u)^{2}\left(1+\int_{\Omega}(1-u)^{-1} d x\right)^{2}}, & x \in \Omega, t>0, \\ u(x, t)=0, & x \in \partial \Omega, t>0, \\ u(x, 0)=u_{0}(x), & x \in \bar{\Omega} .\end{cases}
$$

It is easy to see that problem (P) admits an energy functional of the form

$$
E[u](t) \equiv E(t)=\frac{1}{2} \int_{\Omega}|\nabla u|^{2} d x+\frac{\lambda}{\left(1+\int_{\Omega}(1-u)^{-1} d x\right)},
$$

since

$$
\frac{d E}{d t}=-\int_{\Omega} u_{t}^{2}(x, t) d x \leq 0 .
$$

Hence we have that $E(t)$ is decreasing in time.

The following theorem shows that the solution of problem (P) quenches in finite time for certain initial data.

Theorem 2.1 ([18]). There exist initial data with small initial energy such that the corresponding solution of problem $(\mathrm{P})$ quenches in finite time, if $\lambda$ is chosen sufficiently large.

Proof. Suppose that the problem (P) has a global-in-time (classical) solution $u$. Set

$$
A(t)=\int_{\Omega} u^{2}(x, t) d x .
$$

Multiplying equation (1.1) by $u$ and integrating by parts over $\Omega$, we derive

$$
\frac{1}{2} A^{\prime}(t)=-\int_{\Omega}|\nabla u|^{2} d x+\lambda \frac{\int_{\Omega} \frac{u}{(1-u)^{2}} d x}{\left(1+\int_{\Omega}(1-u)^{-1} d x\right)^{2}} .
$$

Using (2.1) and (2.2), it follows that

$$
\frac{1}{2} A^{\prime}(t) \geq-2 E(0)+\lambda \frac{2+\int_{\Omega} \frac{2-u}{(1-u)^{2}} d x}{\left(1+\int_{\Omega}(1-u)^{-1} d x\right)^{2}} .
$$

On the other hand, using Hölder's and Young's inequalities, we can deduce that

$$
\left(1+\int_{\Omega} \frac{d x}{1-u}\right)^{2} \leq 2+2|\Omega| \int_{\Omega} \frac{d x}{(1-u)^{2}} .
$$


Combining (2.3) and (2.4) yields

$$
\frac{1}{2} A^{\prime}(t) \geq-2 E(0)+\lambda \frac{2+\int_{\Omega} \frac{2-u}{(1-u)^{2}} d x}{2+2|\Omega| \int_{\Omega} \frac{d x}{(1-u)^{2}}} .
$$

Using the fact that $u<1$, we have

$$
\int_{\Omega} \frac{2-u}{(1-u)^{2}} d x \geq \int_{\Omega} \frac{d x}{(1-u)^{2}}
$$

and consequently we derive that

$$
\frac{1}{2} A^{\prime}(t) \geq-2 E(0)+q(|\Omega|) \lambda, \quad q(|\Omega|):= \begin{cases}1, & |\Omega| \leq 1 / 2 \\ 1 /(2|\Omega|), & |\Omega|>1 / 2 .\end{cases}
$$

This implies that

$$
A(t) \geq 2(q(|\Omega|) \lambda-2 E(0)) t+A(0) .
$$

It follows from (2.5) that $A(t) \rightarrow \infty$ as $t \rightarrow \infty$, provided that

$$
E(0)<q(|\Omega|) \lambda / 2 \text {. }
$$

But, $A(t) \leq|\Omega|$ for all $t>0$, a contradiction. The theorem follows.

Remark 2.2. Let $N=1, \Omega=(0,1 / 2)$, and

$$
u_{0}(x)= \begin{cases}4 \delta x, & 0 \leq x \leq 1 / 4 \\ 4 \delta(1 / 2-x), & 1 / 4 \leq x \leq 1 / 2\end{cases}
$$

where $0<\delta<1$. Then we have

$$
E(0)=4 \delta^{2}+\frac{\lambda}{g(\delta)}, \quad g(\delta):=1-\frac{1}{2 \delta} \ln (1-\delta) .
$$

Note that $g(\delta) \rightarrow+\infty$ as $\delta \rightarrow 1^{-}$. Hence condition (2.6) is satisfied if $\delta$ is sufficiently close to 1 and $\lambda$ is sufficiently large.

Remark 2.3. Notice that Theorem 2.1 is not applicable to the case when $u_{0} \equiv 0$. The quenching criterion for zero initial data is still open.

\subsection{With inertia $(\varepsilon=1)$ : hyperbolic case}

In this subsection, we consider the case with inertia so that the problem is of hyperbolic type. We leave $\alpha$ free and normalize $\Omega$ so that $|\Omega|=1$. Then our problem becomes the following initial-boundary value problem:

$$
\begin{cases}u_{t t}+u_{t}=\Delta u+\frac{\lambda h^{2}(u)}{[1+\alpha I(u)]^{2}}, & x \in \Omega, t>0, \\ u(x, t)=0, & x \in \partial \Omega, t>0, \\ u(x, 0)=u_{0}(x), \quad u_{t}(x, 0)=u_{1}(x), & x \in \bar{\Omega} .\end{cases}
$$


Hereafter, we denote

$$
h(u):=\frac{1}{1-u}, \quad I(u)(t):=\int_{\Omega} h(u(x, t)) d x, \quad \Psi(u)(t)=\frac{1}{1+\alpha I(u)(t)} .
$$

Define

$$
E(t)=\int_{\Omega}\left[\frac{1}{2} u_{t}^{2}+\frac{1}{2}|\nabla u|^{2}\right] d x+\frac{\lambda}{\alpha} \Psi(u)(t) .
$$

Then it is easy to check that

$$
\frac{d E}{d t}(t)=-\int_{\Omega} u_{t}^{2} d x \leq 0
$$

and hence $E(t) \leq E(0)$ for $t>0$.

First, we derive a quenching criterion for the case $u_{0} \not \equiv 0$. For a general domain $\Omega \subset \mathbb{R}^{N}$ with $|\Omega|=1$, we have the following quenching criterion (cf. [19]).

Theorem 2.4. Suppose that the initial function $u_{0}$ satisfies

$$
I\left(u_{0}\right)>\frac{4 \alpha^{2}+2-\alpha}{\alpha(2+\alpha)}, \text { if } 0<\alpha \leq 1 / 2 ; \quad I\left(u_{0}\right)>4-\frac{1}{\alpha} \text {, if } \alpha>1 / 2 .
$$

Then there exists a positive constant $\lambda^{+}=\lambda^{+}\left(\alpha ; u_{0}, u_{1}\right)$ such that the solution of (2.7) quenches in finite time, if either $\lambda>\lambda^{+}\left(\alpha ; u_{0}, u_{1}\right)$, or

$$
\lambda=\lambda^{+}\left(\alpha ; u_{0}, u_{1}\right) \text { and }\left\|u_{0}\right\|^{2}+2 \int_{\Omega} u_{0}(x) u_{1}(x) d x>1 .
$$

Proof. Suppose that $|u|<1$ for $(x, t) \in \bar{\Omega} \times[0, \infty)$. Following [18], we set

$$
A(t)=\int_{\Omega} u^{2}(x, t) d x .
$$

Then we compute that

$$
A^{\prime}(t)=2 \int_{\Omega} u u_{t} d x, \quad A^{\prime \prime}(t)=2 \int_{\Omega} u u_{t} d x+2 \int_{\Omega} u_{t}^{2} d x .
$$

It follows from (2.7) and an integration by parts that

$$
A^{\prime \prime}(t)+A^{\prime}(t)=-2 \int_{\Omega}|\nabla u|^{2} d x+2 \int_{\Omega} u_{t}^{2} d x+2 \lambda \frac{\int_{\Omega} u h^{2}(u) d x}{[1+\alpha I(u)]^{2}} .
$$

Using (2.8) and $h(u)=1 /(1-u)$, we can deduce that

$$
A^{\prime \prime}(t)+A^{\prime}(t)=4 \int_{\Omega} u_{t}^{2} d x-4 E(t)+2 \lambda \frac{2+\alpha \int_{\Omega}(2-u) h^{2}(u) d x}{\alpha[1+\alpha I(u)]^{2}} .
$$

Moreover, using Young's inequality and Hölder's inequality, we have

$$
[1+\alpha I(u)]^{2} \leq 2\left[1+\alpha^{2} I(u)^{2}\right] \leq 2+2 \alpha^{2} \int_{\Omega} h^{2}(u) d x .
$$


Since $E(t) \leq E(0)$ for $t \geq 0$ and $|u|<1$ for $(x, t) \in \bar{\Omega} \times[0, \infty)$, we end up with the following inequality

$$
A^{\prime \prime}(t)+A^{\prime}(t) \geq-4 E(0)+\frac{\lambda}{\alpha} \theta\left(\int_{\Omega} h^{2}(u) d x\right),
$$

where $\theta(z):=(2+\alpha z) /\left(1+\alpha^{2} z\right)$ for $z \geq 1$. Note that $\int_{\Omega} h^{2}(u) d x \geq 1$.

Since

$$
\theta^{\prime}(z)=\alpha(1-2 \alpha) /\left(1+\alpha^{2} z\right)^{2},
$$

we see that $\theta(z) \geq \theta(1)=(2+\alpha) /\left(1+\alpha^{2}\right)$ for all $z \geq 1$, if $\alpha \in(0,1 / 2]$, while $\theta(z) \geq \theta(+\infty)=1 / \alpha$ for all $z \geq 1$, if $\alpha>1 / 2$. It follows from (2.11) that

$$
A^{\prime \prime}(t)+A^{\prime}(t) \geq Q(\lambda, \alpha)
$$

where

$$
Q(\lambda, \alpha):= \begin{cases}-4 E(0)+\lambda(2+\alpha) /\left[\alpha\left(1+\alpha^{2}\right)\right], & 0<\alpha \leq 1 / 2, \\ -4 E(0)+\lambda / \alpha^{2}, & \alpha>1 / 2 .\end{cases}
$$

Integrating (2.12) twice and using the fact that $A(t)<1$ for all $t \geq 0$, it follows that

$$
A(t) \geq \frac{1}{2} Q(\lambda, \alpha) t^{2}+\left[A(0)+A^{\prime}(0)-1\right] t+A(0), \forall t \geq 0 .
$$

Recall that

$$
E(0)=\left(\left\|\nabla u_{0}\right\|^{2}+\left\|u_{1}\right\|^{2}\right) / 2+\lambda /\left\{\alpha\left[1+\alpha I\left(u_{0}\right)\right]\right\} .
$$

Hereafter $\|\cdot\|$ denotes the $L^{2}$ norm. Then, under the assumption (2.9), for any fixed $\alpha>0$ we have that $Q(\lambda, \alpha)$ is strictly increasing in $\lambda, Q(0, \alpha)<0$ and $Q(\infty, \alpha)=\infty$. Hence there is a unique positive constant $\lambda^{+}$depending on $\alpha, u_{0}, u_{1}$ such that $Q\left(\lambda^{+}, \alpha\right)=0$. Therefore, if $\lambda>\lambda^{+}(\alpha)$, then $Q(\lambda, \alpha)>0$. It follows from (2.13) that $A(t) \rightarrow \infty$ as $t \rightarrow \infty$, a contradiction. On the other hand, if $\lambda=\lambda^{+}(\alpha)$, then $Q(\lambda, \alpha)=0$. But, the condition (2.10) implies that $A(0)+$ $A^{\prime}(0)>1$. Hence we also obtain from (2.13) that $A(t) \rightarrow \infty$ as $t \rightarrow \infty$, a contradiction. This completes the proof of the theorem.

We remark that Theorem 2.4 is not applicable to the case when $u_{0} \equiv 0$. However, physically we should have the touch down when the applied voltage is sufficiently high. In the following, we shall give some conditions to ensure the touch-down for zero initial data for one dimensional case.

For the case of zero initial and boundary conditions, we let $v=e^{t / 2} u$. Then, for $N=1$ and $\Omega=(0,1), v$ satisfies

$$
\begin{cases}v_{t t}-v_{x x}=\frac{1}{4} v+\frac{\lambda e^{t / 2} h^{2}\left(e^{-t / 2} v\right)}{\left[1+\alpha I\left(e^{-t / 2} v\right)\right]^{2}}, & 0<x<1, t>0, \\ v(0, t)=v(1, t)=0, & t>0, \\ v(x, 0)=v_{t}(x, 0)=0, & 0 \leq x \leq 1 .\end{cases}
$$


By applying a Picard iteration with the help of the domain of dependence for 1D wave equation and the Duhamel principle, we have the following lemma (cf. [27, 19]).

Lemma 2.5. For $\lambda \gg 1$, there exists $t_{0} \in(0,1 / 2]$ such that the solution $v$ of (2.14) satisfies

$$
v(x, t)=V(t)=\max _{x \in[0,1 / 2]} v(x, t)
$$

in the $\operatorname{set}\left\{(x, t) \mid t \leq x \leq 1 / 2,0 \leq t \leq t_{0}\right\}$.

Indeed, the time $t_{0}$, as a function of $\lambda$, is defined so that the solution $V$ to the equation

$$
V(t)=\int_{0}^{t}(t-\tau)\left\{\frac{1}{4} V(\tau)+\lambda e^{\tau / 2} h^{2}\left(e^{-\tau / 2} V(\tau)\right) /\left[1+\alpha I\left(e^{-\tau / 2} V(\tau)\right)\right]^{2}\right\} d \tau .
$$

satisfies $e^{-t / 2} V(t)<1$ for $t \in\left[0, t_{0}\right)$ and $e^{-t_{0} / 2} V\left(t_{0}\right)=1$. Note that the function $W(t):=e^{-t / 2} V(t)$ satisfies

$$
W^{\prime \prime}+W^{\prime}=\frac{\lambda}{(1+\alpha-W)^{2}}, t>0 ; \quad W(0)=W^{\prime}(0)=0 .
$$

It is trivial that $t_{0}(\lambda)$ decreases as $\lambda$ increases such that $t_{0}\left(0^{+}\right)=\infty$ and $t_{0}(+\infty)=0$. Furthermore, we have the estimate

$$
t_{0}(\lambda) \leq 1 / 2, \quad \text { if } \lambda \geq \hat{\lambda}:=\frac{(1+\alpha)^{2}}{1 / \sqrt{e}-1 / 2}
$$

To see this, we assume that $t_{0}(\lambda)>1 / 2$ for some $\lambda \geq \hat{\lambda}$. Then $W(t)<1$ for all $t \leq 1 / 2$. We see that

$$
\left(e^{t} W^{\prime}\right)^{\prime} \geq \frac{\lambda e^{t}}{(1+\alpha)^{2}}, \forall t \leq 1 / 2
$$

This implies that

$$
W(t) \geq \frac{\lambda}{(1+\alpha)^{2}}\left(e^{-t}+t-1\right), \forall t \leq 1 / 2,
$$

and, in particular, $W(1 / 2) \geq 1$, a contradiction. Hence (2.16) is derived.

This lemma gives the following quenching criterion for zero initial data.

Theorem 2.6 ([19]). The solution $u$ to (2.7) with $u_{0}=u_{1}=0$ quenches in a finite time $T \leq 1 / 2$, provided that $\lambda \geq \hat{\lambda}$.

Note that the solution $u$ to (2.7) with $u_{0}=u_{1}=0$ is symmetric with respect to $x=1 / 2$, by the uniqueness of solution. 


\section{Steady states}

In this section, we assume that $\alpha=1$ (after a rescaling). Then the steady states of (1.1) and (1.2) are solutions to the problem

$$
\Delta w+\frac{\lambda}{K(1-w)^{2}}=0, x \in \Omega, \quad w=0, x \in \partial \Omega,
$$

where

$$
K=K(w):=\left(1+\int_{\Omega} \frac{d x}{1-w}\right)^{2}
$$

Note that we always have $0<w<1$ in $\Omega$ for a (classical) solution of (3.1).

By setting

$$
\mu=\frac{\lambda}{K}=\frac{\lambda}{\left(1+\int_{\Omega} \frac{d x}{1-w}\right)^{2}},
$$

then (3.1) is transformed to

$$
\Delta w+\mu(1-w)^{-2}=0 \quad x \in \Omega, \quad w=0 \quad x \in \partial \Omega .
$$

Problems (3.1) and (3.3) are equivalent through (3.2), i.e., $w$ is a solution of (3.1) corresponding to $\lambda$ if and only if $w$ satisfies (3.3) for $\mu=\lambda / K$.

Some structure of steady states of (3.3) is known. Indeed, for any $N$, we recall from [15] that there exists a positive constant $\mu^{*}$ such that a solution of (3.3) exists, if $\mu<\mu^{*}$, and no solutions of (3.3) exist if $\mu>\mu^{*}$. Moreover, the minimal solution, denoted by $w_{\mu}$, of (3.3) is ordered in the sense that

$$
0<w_{\mu_{1}}(x)<w_{\mu_{2}}(x)<1 \quad \text { for } x \in \Omega, \quad \text { if } 0<\mu_{1}<\mu_{2}<\mu^{*} \text {. }
$$

Motivated by the work of Deng [2], we have the following theorem for 1D problem.

Theorem 3.1 ([17]). Suppose that $N=1$. Then there exists a positive constant $\lambda^{*}$ such that there are no steady state solutions if $\lambda>\lambda^{*}$; there is exactly one steady state solution if $\lambda=\lambda^{*}$; and there are two steady state solutions if $\lambda<\lambda^{*}$.

Note that the structure of $1 \mathrm{D}$ steady states of (3.1) is the same as that of local problem (3.3) (cf. [28]). We also refer the reader to [1, 2, 3, 16, 20] for the steady states structures of other nonlocal parabolic problems.

In the following, we shall focus on the case $N \geq 2$. For a general smooth domain $\Omega$, using the monotonicity property (3.4) we can prove the following existence theorem. 
Theorem 3.2 ([18]). There exists a (classical) steady-state solution of $(\mathrm{P})$ if $\lambda \in\left(0,(1+|\Omega|)^{2} \mu^{*}\right)$.

Moreover, we have

Theorem 3.3 ([18]). Let $\Omega$ be a strictly star-shaped domain of $\mathbb{R}^{N}, N \geq 2$. Then problem (3.1) has no solution if

$$
\lambda>\frac{N(1+|\Omega|)^{4}}{2 \beta|\Omega|^{2}}
$$

where $\beta$ is a positive constant depending on $\Omega$. Moreover, for $N \geq 2$ there exists $\lambda_{*}>0$ such that problem (3.1) has a unique solution for $0<\lambda<\lambda_{*}$.

When $\Omega$ is the unit ball, then the solution of (3.1) is radial symmetric, [10], i.e., $w(x)=$ $w(r)$, where $r=|x|$ and (3.1) is reduced to

$$
\begin{aligned}
& \left(r^{N-1} w_{r}\right)_{r}+\frac{\lambda r^{N-1}}{K(1-w)^{2}}=0 \text { for } r \in(0,1), \\
& w(1)=0, \quad w_{r}(0)=0 .
\end{aligned}
$$

Furthermore, $K$ has the form

$$
K=\left(1+N \omega_{N} \int_{0}^{1} \frac{r^{N-1}}{1-w} d r\right)^{2}
$$

The related local problem is

$$
\begin{aligned}
& \left(r^{N-1} w_{r}\right)_{r}+\frac{\mu r^{N-1}}{(1-w)^{2}}=0 \quad \text { for } r \in(0,1), \\
& w(1)=0, \quad w_{r}(0)=0,
\end{aligned}
$$

where $\mu=\lambda / K$.

Indeed, when $\Omega$ is the unit ball of $\mathbb{R}^{N}, N \geq 2$, it is proved by Joseph and Lundgren in [25] that the set of steady states of (3.7)-(3.8) is homeomorphic to $\mathbb{R}$ and has end points $(0,0)$ and $\left(\hat{\mu}, 1-|x|^{2 / 3}\right)$, where $\hat{\mu}:=[4+6(N-2)] / 9$. Moreover, when $2 \leq N \leq 7$ the solution curve $(\mu(s), w(\cdot, s)), s \in \mathbb{R}$, of (3.3) bends infinitely many times around the singular point $\left(\hat{\mu}, 1-r^{2 / 3}\right)$. Whereas for $N \geq 8$ the solution curve terminates at $\left(\hat{\mu}, 1-r^{2 / 3}\right)$ and no bendings occur.

The following theorem shows that the structure of steady states of local problem is preserved, namely, the structures of steady states of local and nonlocal problems are the same. Due to the nonlocal feature, the proof needs a more delicate analysis of the orbit $\mathscr{O}$ (defined in [25]), especially, for the case $2 \leq N \leq 7$.

Theorem 3.4 ([18]). If $N \geq 2$, then the set of steady states of (3.5)-(3.6) is homeomorphic to $\mathbb{R}$ and has end points $(0,0)$ and $\left(\widehat{\lambda}, 1-r^{2 / 3}\right)$, where

$$
\widehat{\lambda}=\Lambda\left(1+\frac{N \omega_{N}}{\gamma}\right)^{2}, \quad \Lambda:=\frac{2}{3}(N-4 / 3)=\hat{\mu}, \quad \gamma=N-2 / 3 .
$$


Moreover, if $2 \leq N \leq 7$, then $\mathscr{C}_{r}$ bends infinitely many times with respect to $\lambda$ around $\hat{\lambda}$ and there exist two positive constants $\lambda^{*}$ and $\lambda_{*}$ with $0<\lambda_{*}<\widehat{\lambda}<\lambda^{*}$ such that problem (3.5)-(3.6) has

1. a unique solution for $0<\lambda<\lambda_{*}$ and $\lambda=\lambda^{*}$,

2. a finite number of solutions for $\lambda \in\left(\lambda_{*}, \lambda^{*}\right)$ and $\lambda \neq \hat{\lambda}$,

3. infinite number of solutions for $\lambda=\hat{\lambda}$,

4. no solutions for $\lambda>\lambda^{*}$.

On the other hand, no bending occurs in the case of $N \geq 8$, i.e., problem (3.5)-(3.6) has a unique solution for any $0<\lambda<\widehat{\lambda}=\lambda^{*}$ and no solution for $\lambda \geq \widehat{\lambda}=\lambda^{*}$.

\section{Global existence}

It is well-known that the steady states of the evolution problem play an important role in the study of its long-time behavior and/or finite time singular behavior. However, due to the nonlocal nonlinearity, we do not have the comparison principle for our problem. Therefore, we use another approach to tackle the global existence of solutions here.

\subsection{Without inertia $(\varepsilon=0)$ : parabolic case}

Given $\left.\lambda \in\left(0,(1+|\Omega|)^{2}\right) \mu^{*}\right)$, there exists a minimal steady-state solution of (3.3), denoted by $w_{\mu}$, with $\mu=\lambda /(1+|\Omega|)^{2}$. Then we have the following theorem on the global existence for small values of $\lambda$.

Theorem 4.1 ([18]). For any $\lambda \in\left(0,(1+|\Omega|)^{2} \mu^{*}\right)$, let $\mu=\lambda /(1+|\Omega|)^{2}$. Then the solution of problem $(\mathrm{P})$ with $u_{0} \leq w_{\mu}$ exists globally in time.

Proof. Let $v$ be the solution to the local problem (LP):

$$
\left\{\begin{array}{l}
v_{t}=\Delta v+\mu(1-v)^{-2}, x \in \Omega, t>0 \\
v=0 \text { on } \partial \Omega \\
v=u_{0} \text { for } t=0
\end{array}\right.
$$

Since $u_{0} \leq w_{\mu}$, by comparison, $v \leq w_{\mu}$ and $v$ exists globally in time. Moreover, since $u$ is a sub-solution of the problem (LP), by comparison, we have $u \leq v$. This implies that $u$ exists globally and the theorem follows.

Note that the solution $\underline{v}$ to (LP) with $u_{0} \equiv 0$ exists globally such that $\underline{v}_{t}>0$ and $\underline{v} \leq v$. Hence $\underline{v}$ converges to the minimal steady state $w_{\mu}$ as $t \rightarrow \infty$. This also implies that $v \rightarrow w_{\mu}$ 
as $t \rightarrow \infty$. It would be interesting to see whether $u$ tends to some steady state for general dimension $N$.

\subsection{With inertia $(\varepsilon=1)$ : hyperbolic case}

We first have the following criterion of the global existence for zero initial data.

Theorem 4.2 ([19]). Suppose that $N=1$ and let $\Omega=(0,1)$. If $u_{0}=u_{1}=0$ and

$$
0<\lambda<2(1+\alpha) \max _{0 \leq \delta \leq 1}(1-\delta)(\alpha+\delta),
$$

then the solution $u=u(x, t ; \lambda)$ of $(2.7)$ exists globally in time.

For the case of non-zero initial data such that $\left\|u_{0}^{\prime}\right\|^{2}+\left\|u_{1}\right\|^{2}<4$, we have the global existence if

$$
0<\lambda<\left[4-\left(\left\|u_{0}^{\prime}\right\|^{2}+\left\|u_{1}\right\|^{2}\right)\right] \alpha / 2 .
$$

Indeed, suppose that $u \leq M<1$ in $[0,1] \times[0, T]$. Then we have

$$
\Psi(u)(t) \geq \frac{1}{1+\alpha /(1-M)} \text { for all } t \in[0, T] .
$$

Using $E(t) \leq E(0)$,

$$
4 u^{2} \leq \int_{0}^{1} u_{x}^{2} d x
$$

and $\Psi(u) \leq 1$, we obtain

$$
4 M^{2}+\frac{2 \lambda}{\alpha} \frac{1-M}{1+\alpha-M} \leq\left\|u_{0}^{\prime}\right\|^{2}+\left\|u_{1}\right\|^{2}+\frac{2 \lambda}{\alpha}
$$

for all $t \in[0, T]$. If $u$ quenches in finite time $T$, by letting $M \rightarrow 1$ in (4.2), we obtain a contradiction to (4.1). Hence we have the global existence when the condition (4.1) is imposed.

\section{Open problems}

Due to the lack of comparison principle, little is known for our nonlocal problem. Although we have provided some quenching criteria in section 2, our results are far from complete. For example, as mentioned in Remark 2.3, the quenching criterion is still open for zero initial data in the parabolic case. Also, the stabilization for the global solution of the nonlocal problem is still open.

The second question is about the location of touch-down. Let $T<\infty$ be the quenching time. Mathematically, a point $x_{0}$ is a quenching point (touch-down point), if there is a sequence $\left\{\left(x_{n}, t_{n}\right)\right\}$ in $\bar{\Omega} \times[0, T)$ such that $x_{n} \rightarrow x_{0}, t_{n} \uparrow T$ and $u\left(x_{n}, t_{n}\right) \rightarrow 1$ as $n \rightarrow \infty$. For $N=1$ 
and $\Omega=(-1,1)$, it is proved in [17] that 0 is the only quenching point of $(\mathrm{P})$, provided that the initial data $u_{0}$ satisfies

$$
u_{0}(-x)=u_{0}(x), u_{0}^{\prime}(x) \geq 0, x \in[0,1], u_{0} \leq 1-c_{0}, u_{0}^{\prime \prime} \leq-c_{0}
$$

for some constant $c_{0} \in(0,1)$. It would be important to determine the set of quenching points when the solution quenches in finite time.

The next question is to determine the time asymptotic rate when quenching occurs. This is also called the quenching rate. For the quenching rates of some local problems, we refer the reader to $[13,7,14]$. To the author's knowledge, the only known quenching rate for a nonlocal problem is given in [16]. In [16], the nonlocal term in the model is given by

$$
\lambda\left(\int_{0}^{1} u(x, t) d x\right)(1-u)^{-\beta}, \beta>0
$$

in which the comparison principle can be applied. It is proved in [16] that the quenching rate is the same as the local problem without the integral term, namely, $1-u \sim(T-t)^{1 /(\beta+1)}$ as $t \uparrow T$, the so-called self-similar rate. We are not sure whether the quenching rate for the problem $(\mathrm{P})$ is self-similar.

Finally, we mention the question of spatially quenching profile, namely, to determine the profile of $u\left(x, T^{-}\right)$for $x \in \Omega$, or, at least near the quenching point. We refer the reader to [8] for the quenching profile of the local problem.

\section{References}

[1] G.-J. Chen and J.-S. Guo, Critical length for a quenching problem with nonlocal singularity, Methods and Applications of Analysis, 5 (1998), 185-194.

[2] K. Deng, Dynamical behavior of solutions of a semilinear heat equation with nonlocal singularity, SIAM J. Math. Anal., 26 (1995), 98-111.

[3] K. Deng, M.K. Kwong, and H.A. Levine, The influence of nonlocal nonlinearities on the long time behavior of solutions of Burgers' equation, Quart. Appl. Math., 50 (1992), 173-200.

[4] P. Esposito, N. Ghoussoub and Y. Guo, Compactness along the first branch of unstable solutions for an elliptic problem with a singular nonlinearity, Comm. Pure Appl. Math., 60 (2007), 1731-1768.

[5] P. Esposito, Compactness of a nonlinear eigenvalue problem with a singular nonlinearity, Comm. Contemp. Math., 10 (2008), 17-45.

[6] P. Esposito and N. Ghoussoub, Uniqueness of solutions for an elliptic equation modeling MEMS, Methods Appl. Anal., 15 (2008), 341-354.

[7] M. Fila and J. Hulshof, A note on the quenching rate, Proc. Amer. Math. Soc., 112 (1991), 473-477.

[8] S. Filippas, J.-S. Guo, Quenching profiles for one-dimensional semilinear heat equations, Quart. Appl. Math., 51 (1993), 713-729.

[9] G. Flores, G. Mercado, J.A. Pelesko and N. Smyth, Analysis of the dynamics and touchdown in a model of electrostatic MEMS, SIAM J. Appl. Math., 67 (2007), 434-446.

[10] B. Gidas, W.-M. Ni, and L. Nirenberg, Symmetry and related properties via the maximum principle, Comm. Math. Phys., 68 (1979) 209-243. 
[11] N. Ghoussoub and Y. Guo, On the partial differential equations of electrostatic MEMS devices: stationary case, SIAM J. Math. Anal., 38 (2007), 1423-1449.

[12] N. Ghoussoub and Y. Guo, On the partial differential equations of electrostatic MEMS devices II: dynamic case, Nonlinear Diff. Eqns. Appl., 15 (2008), 115-145.

[13] J.-S. Guo, On the quenching behavior of the solution of a semilinear parabolic equation, J. Math. Anal. Appl., 151 (1990), 58-79.

[14] J.-S. Guo, On the quenching rate estimate, Quart. Appl. Math., 49 (1991), 747-752.

[15] J.-S. Guo, Quenching problem in nonhomogeneous media, Differential and Integral Equations, 10 (1997), 1065-1074.

[16] J.-S. Guo, Quenching behavior for the solution of a nonlocal semilinear heat equation, Differential and Integral Equations, 13 (2000), 1139-1148.

[17] J.-S. Guo, B. Hu, and C.-J. Wang, A nonlocal quenching problem arising in micro-electro mechanical systems, Quarterly Appl. Math., 67 (2009), 725-734.

[18] J.-S. Guo, N.I. Kavallaris, On a nonlocal parabolic problem arising in electrostatic MEMS control, Discrete Contin. Dyn. Syst., 32 (2012), 1723-1746.

[19] J.-S. Guo, B.-C. Huang, Hyperbolic quenching problem with damping in the micro-electro mechanical system device, Discrete Contin. Dyn. Syst. Series B, 19 (2014), 419-434.

[20] J.-S. Guo and T.-M. Hwang, On the steady states of a nonlocal semilinear heat equation, Dynamics of Continuous, Discrete and Impulsive Systems, Series A: Mathematical Analysis, 8 (2001), 53-68.

[21] Y. Guo, Z. Pan, and M.J. Ward, Touchdown and pull-in voltage behavior of a MEMS device with varying dielectric properties, SIAM J. Appl. Math., 166 (2006), 309-338.

[22] Y. Guo, On the partial differential equations of electrostatic MEMS devices III: refined touchdown behavior, J. Diff. Eqns., 244 (2008), 2277-2309.

[23] Y. Guo, Global solutions of singular parabolic equations arising from electrostatic MEMS, J. Diff. Eqns., 245 (2008), 809-844.

[24] Z. Guo and J. Wei, Asymptotic Behavior of touch-down solutions and global bifurcations for an elliptic problem with a singular nonlinearity, Comm. Pure Appl. Anal., 7 (2008), 765-786.

[25] D.D. Joseph and T.S. Lundgren, Quasilinear Dirichlet problems driven by positive sources, Arch. Rational Mech. Anal., 49 (1973), 241-269.

[26] N.I. Kavallaris, T. Miyasita, and T. Suzuki, Touchdown and related problems in electrostatic MEMS device equation, Nonlinear Diff. Eqns. Appl. 15, (2008), 363-385.

[27] N.I. Kavallaris, A.A. Lacey, C.V. Nikolopoulos, and D.E. Tzanetis, A hyperbolic non-local problem modelling MEMS technology, Rocky Mountain J. Math., 41 (2011), 505-534.

[28] H.A. Levine, Quenching, nonquenching, and beyond quenching for solution of some parabolic equations, Ann. Mat. Pura Appl., 155 (1989), 243-260.

[29] J.A. Pelesko and A.A. Triolo, Nonlocal problems in MEMS device control, J. Engrg. Math., 41 (2001), 345-366.

[30] J.A. Pelesko and D.H. Bernstein, Modeling MEMS and NEMS, Chapman Hall and CRC Press, 2002.

Department of Mathematics, Tamkang University, Tamsui, New Taipei City 25137, Taiwan.

E-mail: jsguo@mail.tku.edu.tw 